Applied and

NISTIR 4364

Computational

Mathematics

Division

Center for Computing and Applied Mathematics

Effect of a Crystal-Melt Interface on Taylor-Vortex Flow with Buoyancy

G.B. McFadden, B. T. Murray, S.R. Coriell, M. E. Glicksman, and M. E. Selleck

July 1990

$-\mathrm{QC}$

100

.056

\#4364

1990

C. 2
U.S. DEPARTMENT OF COMMERCE National Institute of Standards and Technology Gaithersburg, MD 20899 
NATIONAL INSTITUTE OF STANDARDS \&

TECHIOLOGY

Research Information Center

Gaithersburg, MD 20899

\section{DATE DUE}

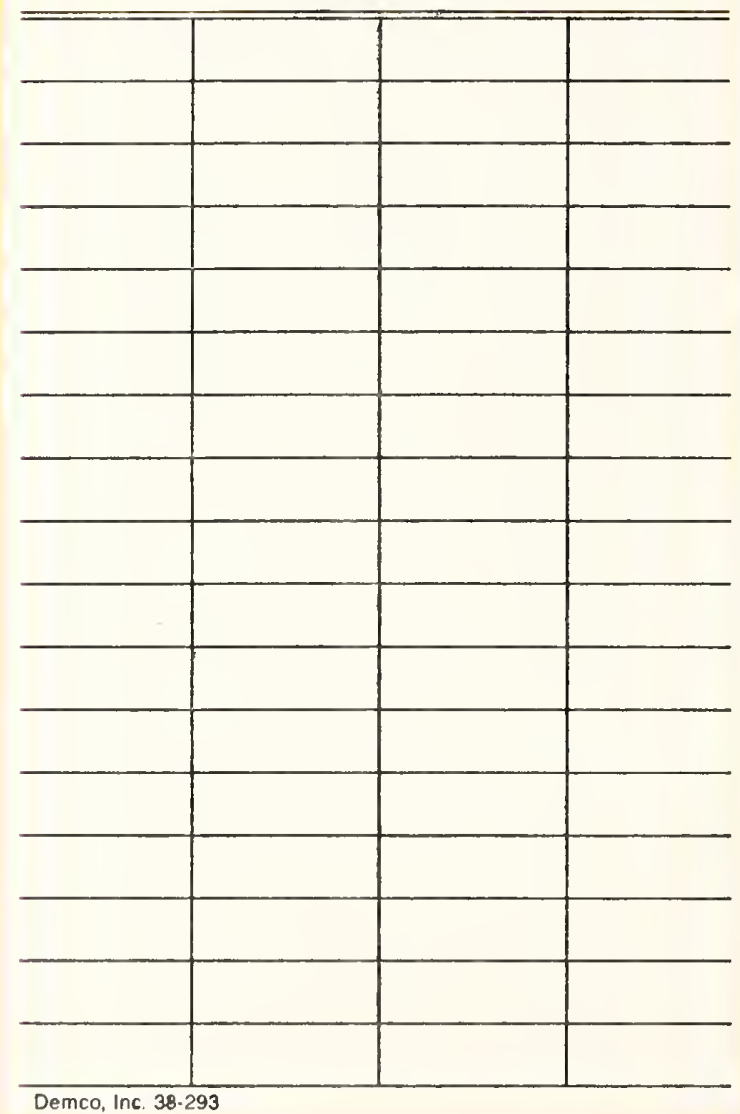


EFFECT OF A CRYSTAL-MELT INTERFACE ON TAYLOR-VORTEX FLOW WITH BUOYANCY
G. B. McFadden

B. T. Murray

S. R. Coriell

M. E. Glicksman

M. E. Selleck

U.S. DEPARTMENT OF COMMERCE National Instituto of Standards and Tochnology Center for Computing and Applied Mathomatles Applled and Computational Mathematice Divislon Galthorburg, MD 20899

July 1990

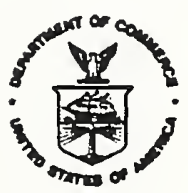

U.S. DEPARTMENT OF COMMERCE Robert A Mosbacher, secretary NATIONAL INSTTUTE OF STANDARDS AND TECHNOLOOY

John W. Lyons, Director 



\title{
Effect of a Crystal-Melt Interface on Taylor-Vortex Flow with Buoyancy
}

\author{
G. B. McFadden, B. T. Murray, and S. R. Coriell \\ National Institute of Standards and Technology \\ Gaithersburg, MD 20899 \\ M. E. Glicksman and M. E. Selleck \\ Department of Materials Engineering \\ Rensselaer Polytechnic Institute \\ Troy, NY 12181
}

\begin{abstract}
During crystal growth from the melt, a fundamental problem is to understand the interaction of the crystal-melt interface with fluid flow in the melt. This problem combines the complexities of the Navier-Stokes equations for fluid flow with the nonlinear behavior of the free boundary representing the crystal-melt interface. Some progress has been made by studying explicit flows that allow a base state corresponding to a one-dimensional crystal-melt interface with solute and/or temperature fields that depend only on the distance from the interface. This allows the strength of the interaction between the flow and the interface to be assessed by a linear stability analysis of the simple base state. The case of a Taylor-Couette flow interacting with a cylindrical crystalline interface is currently being investigated both experimentally and theoretically. We consider the changes in the linear stability of this system produced by density-driven convection generated by the interaction of the density gradients with the gravitational and centripetal acceleration.
\end{abstract}

\section{Introduction}

The study of crystal growth from the liquid or melt phase provides a rich source of free boundary problems, the solutions for which are of great practical interest. (Some descriptions of common crystal growth techniques are given by Hurle and Jakeman in [1] and references therein.) When growing crystals of doped semiconductors or metallic alloys, the concentration of so- . lute at the freezing interface is of special concern [2]. In most applications it is desirable to produce crystals with homogeneous distributions of solute 
throughout the crystal, and great care is taken in the design of the crystal growth apparatus to attempt to control the concentration and thermal fields near the interface. In directional solidification from the melt, for example, an idealized furnace that is free from imperfections would produce a planar crystal-melt interface with one-dimensional temperature and solute fields, allowing solute to be incorporated uniformly in the growing crystal. In reality, even such a planar crystal-melt interface is subject to various instabilities $[3,4]$ which can result in segregation of solute at the interface and produce inhomogeneous distributions of solute in the crystal. In addition to instabilities associated with the interface itself [5], under terrestrial growth conditions it is often difficult to avoid the occurrence of fluid flow in the melt due to natural convection [6]. Such flows are themselves able to cause undesirable segregation of solute [7] and may result in the production of inferior quality crystal. Avoiding natural convection is one of the main motivations for developing the capability of crystal growth under the microgravity conditions available in low earth orbit, where the driving force for natural convection is lower by orders of magnitude. In addition, such an environment allows more precise fundamental experiments on interface dynamics to be performed without the complicating effects of buoyancy-driven convection [8].

The study of the interaction of fluid flow with a crystal-melt interface is thus an area of fundamental importance in materials science, but despite much recent research [9] the understanding of such interactions is fragmentary. The general problem combines the complexities of the Navier-Stokes equations for the fluid flow in the melt with the nonlinear behavior of the free boundary representing the crystal-melt interface. Some progress has been made by studying explicit flows that allow a base state corresponding to a one-dimensional crystal-melt interface with solute and/or temperature fields that depend only on the distance from the interface. This allows the 
strength of the interaction between the flow and the interface to be assessed by a linear stability analysis of the simple base state.

For example, one can examine changes in the morphological stability [5] of the interface in the presence of flow in the melt. Specific flows that have been considered in this way include plane Couette flow $[10,11]$, thermosolutal convection $[7,12]$, plane stagnation flow $[13,14]$, rotating disk flow [15], and the asymptotic suction profile [16]. One can also examine changes in the hydrodynamic stability of a given flow that occur when a rigid bounding surface is replaced by a crystal-melt interface. Examples here include the instabilities associated with Rayleigh-Bénard convection [17], thermosolutal convection $[6,18,19]$, plane Poiseuille flow [20], the asymptotic suction profile [16], thermally-driven flow in an annulus [20,21], and Taylor Couette flow $[22,23]$.

In previous work $[22,23]$ we have described the interaction of a TaylorCouette flow with a cylindrical crystal-melt interface under the assumption that the effects of buoyancy can be neglected. This preliminary work is in support of experimental studies being conducted with succinonitrile; for this material the crystal-melt interface is predicted to have a significant effect on the conditions for marginal stability of the flow. Since large temperature differences in the system are capable of driving natural convection in the melt $[24,25]$, it is desirable to include such effects in our theoretical treatment as well. In this paper we reformulate the problem to include the effects of buoyancy when the axis of the cylinders is aligned with the direction of gravity. This generates a more complicated flow field in the base state, which then is subject to not only centrifugal instabilities but buoyant instabilities as well $[26,27]$. We also include the effects of densitydriven convection produced by the interaction between the radial density gradient and the centripetal acceleration of the azimuthal flow in the base state [28]. Linear stability results are obtained numerically for a typical 


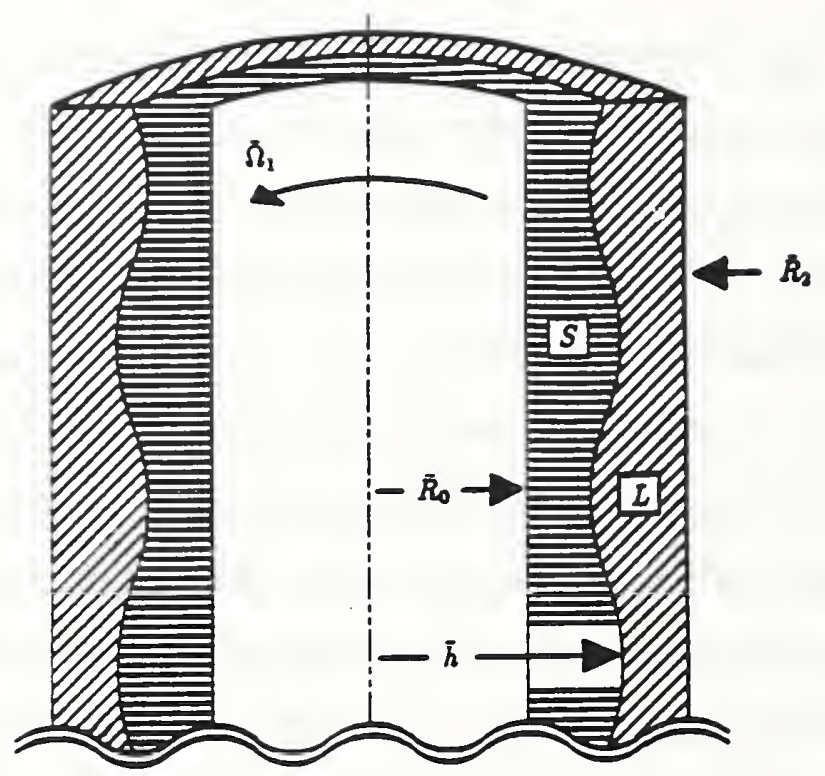

FIG. 1. Schematic diagram of the crystalline inner annulus (labeled "S") surrounded by the liquid phase (labeled " $L$ "). In the base state the unperturbed crystal-melt interface is cylindrical, with $\bar{h}(\bar{z}, \phi, \bar{t})=\bar{R}_{1}$.

experimental configuration. For these conditions we find that the effect of the contribution from the interaction of the radial density gradient with the centripetal acceleration is not significant. The natural convection causes a slight stabilization of the system; however, there is a two-fold increase in the wavelength of the most dangerous disturbance.

\section{Taylor-Couette Flow with Buoyancy}

We consider Taylor-Couette flow $[29,30]$ in the presence of a crystal-melt interface $\bar{r}=\bar{h}(\bar{z}, \phi, \bar{t})$ (overbars will denote dimensional quantities). A cylindrical coordinate system $(r, z, \phi)$ is used. The melt occupies the region $\bar{h}(\bar{z}, \phi, \bar{t})<\bar{r}<\bar{R}_{2}$, and the crystal occupies the region $\bar{R}_{0}<\bar{r}<\bar{h}(\bar{z}, \phi, \bar{t})$ (see Fig. 1); in the unperturbed base state the interface is an infinite cylinder with $\bar{h}=\bar{R}_{1}$. We also consider the convection-diffusion equation for heat transport as well. We consider steady rotation of the system; the outer cylinder is stationary and the inner cylinder and crystal rotate with 
angular velocity $\bar{\Omega}_{1}$. The temperature dependence of the density is taken into account in a Boussinesq approximation [31], which allows convective effects due to the interaction oi the density gradient with the gravitational and centripetal acceleration terms. We assume the axis of the cylinders is aligned with the gravity vector, $-g \hat{z}$, where $\hat{z}$ is the unit vector along the $z$-axis.

The nonlinear dimensional governing equations in the melt are the continuity equation,

$$
\frac{\partial \bar{u}}{\partial \bar{r}}+\frac{\bar{u}}{\bar{r}}+\frac{1}{\bar{r}} \frac{\partial \bar{v}}{\partial \phi}+\frac{\partial \bar{w}}{\partial \bar{z}}=0
$$

the momentum equations,

$$
\begin{gathered}
\bar{\rho}_{0} \frac{D \bar{u}}{D \bar{t}}+\frac{\partial \bar{p}}{\partial \bar{r}}=\mu\left(\bar{\nabla}^{2} \bar{u}-\frac{\bar{u}}{\bar{r}^{2}}-\frac{2}{\bar{r}^{2}} \frac{\partial \bar{v}}{\partial \phi}\right)+\bar{\rho} \frac{\bar{v}^{2}}{\bar{r}} \\
\bar{\rho}_{0} \frac{D \bar{v}}{D \bar{t}}+\frac{1}{\bar{r}} \frac{\partial \bar{p}}{\partial \phi}=\mu\left(\bar{\nabla}^{2} \bar{v}-\frac{\bar{v}}{\bar{r}^{2}}+\frac{2}{\bar{r}^{2}} \frac{\partial \bar{u}}{\partial \phi}\right)-\bar{\rho} \frac{\bar{u} \bar{v}}{\bar{r}} \\
\bar{\rho}_{0} \frac{D \bar{w}}{D \bar{t}}+\frac{\partial \bar{p}}{\partial \bar{z}}=\mu \bar{\nabla}^{2} \bar{w}-\bar{\rho} g
\end{gathered}
$$

and the energy equation

$$
\frac{D \bar{T}}{D \bar{t}}=\kappa \bar{\nabla}^{2} \bar{T}
$$

where

$$
\frac{D}{D \bar{t}}=\frac{\partial}{\partial \bar{t}}+\bar{u} \frac{\partial}{\partial \bar{r}}+\frac{\bar{v}}{\bar{r}} \frac{\partial}{\partial \phi}+\bar{w} \frac{\partial}{\partial \bar{z}}
$$

and

$$
\bar{\nabla}^{2}=\frac{\partial^{2}}{\partial \bar{r}^{2}}+\frac{1}{\bar{r}} \frac{\partial}{\partial \bar{r}}+\frac{1}{\bar{r}^{2}} \frac{\partial^{2}}{\partial \phi^{2}}+\frac{\partial^{2}}{\partial \bar{z}^{2}} .
$$

Here $\bar{u}, \bar{v}$, and $\bar{w}$ are the velocity components in the $\bar{r}, \phi$, and $\bar{z}$ directions, respectively, $\bar{p}$ is the pressure, $\bar{T}$ is the temperature in the melt, $\mu$ is the viscosity coefficient, $\kappa$ is the thermal diffusivity in the melt, and $\bar{\rho}=\bar{\rho}_{0}\left(1-\alpha\left[\bar{T}-\bar{T}_{E}\right]\right)$ is the density, where $\bar{\rho}_{0}$ and $\bar{T}_{E}$ are reference densities and temperatures, and $\alpha$ is the coefficient of thermal expansion. In the 
Boussinesq approximation employed here [28, 31], the temperature variation in the density is neglected in the advection terms on the left hand sides of the momentum equations.

The solid is rotating with an azimuthal velocity $\bar{r} \bar{\Omega}_{1}$, and the temperature in the solid, $\bar{T}_{S}$, obeys

$$
\frac{\partial \bar{T}_{S}}{\partial \bar{t}}+\bar{\Omega}_{1} \frac{\partial \bar{T}_{S}}{\partial \phi}=\kappa_{S} \bar{\nabla}^{2} \bar{T}_{S}
$$

where $\kappa_{S}$ is the thermal diffusivity in the crystal.

At the outer boundary $\bar{r}=\bar{R}_{2}$, we have $\bar{u}=\bar{w}=\bar{v}=0$, and $\bar{T}=\bar{T}_{2}$, where $\bar{T}_{2}$ is the (constant) temperature imposed at the outer cylinder; $\vec{T}_{2}$ is assumed to exceed the melting point $\bar{T}_{m}$ of the material. At the inner boundary $\bar{r}=\bar{R}_{0}$, we have $\bar{T}_{S}=\bar{T}_{0}$, where $\bar{T}_{0}$ is the (constant) temperature imposed at the inner cylinder; $\bar{T}_{0}$ is assumed to lie below $\bar{T}_{m}$. At the interface $\bar{r}=\bar{h}(\bar{z}, \phi, \bar{t})$ the boundary conditions are [6]

$$
\begin{gathered}
\bar{u}=\bar{w}=0, \\
\bar{v}=\bar{h}(\bar{z}, \phi, \bar{t}) \bar{\Omega}_{1}, \\
\bar{T}=\bar{T}_{S}=\bar{T}_{m}-\bar{T}_{m} \Gamma \bar{K}, \\
-L_{V} \frac{\partial \bar{h}}{\partial \bar{t}}=k_{L}\left(\frac{\partial \bar{T}}{\partial \bar{r}}-\frac{1}{\bar{h}^{2}} \frac{\partial \bar{h}}{\partial \phi} \frac{\partial \bar{T}}{\partial \phi}-\frac{\partial \bar{h}}{\partial \bar{z}} \frac{\partial \bar{T}}{\partial \bar{z}}\right)- \\
k_{S}\left(\frac{\partial \bar{T}_{S}}{\partial \bar{r}}-\frac{1}{\bar{h}^{2}} \frac{\partial \bar{h}}{\partial \phi} \frac{\partial \bar{T}_{S}}{\partial \phi}-\frac{\partial \bar{h}}{\partial \bar{z}} \frac{\partial \bar{T}_{S}}{\partial \bar{z}}\right),
\end{gathered}
$$

where $\Gamma$ is a capillary length, $L_{V}$ is the latent heat of fusion per unit volume of crystal, $\bar{K}$ is the mean curvature of the interface, and $k_{L}$ and $k_{S}$ are the thermal conductivities in the liquid and solid, respectively. We have assumed equal densities of crystal and melt, and equal heat capacities in each phase. 


\section{Base State}

The equations admit an annular base state with $\bar{h}(\bar{z}, \phi, \bar{t})=\bar{R}_{1}, \bar{u}=0, \bar{v}=$ $\bar{V}(\bar{r}), \bar{w}=\bar{W}(\bar{r}), \bar{p}=\bar{P}(\bar{r}, \bar{z}), \bar{T}=\bar{\Theta}(\bar{r})$, and $\bar{T}_{S}=\bar{\Theta}_{S}(\bar{r})$. Dimensionless variables may be introduced as follows. The length scale is chosen to be the melt gap width $\bar{L}=\bar{R}_{2}-\bar{R}_{1}$, the time scale is chosen to be $\bar{L}^{2} / \nu$, where $\nu=\mu / \bar{\rho}_{0}$ is the kinematic viscosity, the velocity scale is $\nu / \bar{L}$, the pressure scale is $\overline{\rho_{0}} \nu^{2} / \bar{L}^{2}$, and the deviation of the temperature from its value at the unperturbed interface is measured in units of the temperature difference across the melt, $\Delta T=\bar{T}_{2}-\bar{T}_{m}+\bar{T}_{m} \Gamma / \bar{R}_{1}$. The melt then occupies the region $\eta /(1-\eta)<r<1 /(1-\eta)$, the unperturbed interface is located at $r=\eta /(1-\eta)$, and the crystal occupies the region $\eta_{S} /(1-\eta)<r<\eta /(1-\eta)$, where $\eta=\bar{R}_{1} / \bar{R}_{2}$ and $\eta_{s}=\bar{R}_{0} / \bar{R}_{2}<\eta$. (Dimensionless counterparts to the dimensional variables will lack overbars.)

The resulting dimensionless expressions for the base state variables are as follows. The base azimuthal velocity can be written in the form

$$
V(r)=\frac{-\operatorname{Re} \eta^{2}}{\left(1-\eta^{2}\right)} r+\frac{1}{r} \frac{\operatorname{Re} \eta^{2}}{\left(1-\eta^{2}\right)(1-\eta)^{2}},
$$

where $\operatorname{Re}=\bar{L}^{2} \bar{\Omega}_{1} / \nu$ is the Reynolds number. The solution for the axial velocity that is appropriate to model a closed system with no net axial volume flux,

$$
\int_{R_{1}}^{R_{2}} \bar{r} \bar{W}(\bar{r}) d \bar{r}=0
$$

is given by

$$
W(r)=\frac{-G}{16(1-\eta)^{2}}\left(C\left[\xi^{2}-1+\left(1-\eta^{2}\right) \frac{\ln \xi}{\ln \eta}\right]-4\left(\xi^{2}-\eta^{2}\right) \frac{\ln \xi}{\ln \eta}\right),
$$

where $G=g \alpha \bar{L}^{3} \Delta T / \nu^{2}$ is the Grashof number, $\xi=(1-\eta) r$, and

$$
C=\frac{\left(1-\eta^{2}\right)\left(1-3 \eta^{2}\right)-4 \eta^{4} \ln \eta}{\left(1-\eta^{2}\right)^{2}+\left(1-\eta^{4}\right) \ln \eta}
$$

The dimensionless temperature fields are given by

$$
\Theta(r)=\frac{\ln (\xi / \eta)}{\ln (1 / \eta)}
$$


and

$$
\Theta_{S}(r)=\left(\frac{k_{L}}{k_{S}}\right) \frac{\ln (\xi / \eta)}{\ln (1 / \eta)},
$$

The radial pressure gradient balances the centrifugal force, and the axial pressure gradient is constant.

\section{Linearized equations}

We next consider the dimensionless equations linearized about the steady base state. We Fourier transform the axial and azimuthal coordinates, which introduces the axial wavenumber $a$ and the azimuthal wavenumber $n$, and write

$$
\left(\begin{array}{c}
u(r, z, \phi, t) \\
v(r, z, \phi, t) \\
w(r, z, \phi, t) \\
p(r, z, \phi, t) \\
T(r, z, \phi, t) \\
T_{S}(r, z, \phi, t) \\
h(z, \phi, t)
\end{array}\right)=\left(\begin{array}{c}
0 \\
V(r) \\
W(r) \\
P(r, z) \\
\Theta(r) \\
\Theta_{S}(r) \\
\eta /(1-\eta)
\end{array}\right)+\left(\begin{array}{c}
\hat{u}(r) \\
\hat{v}(r) \\
\hat{w}(r) \\
\hat{p}(r) \\
\hat{\Theta}(r) \\
\hat{\Theta}(r) \\
\hat{h}
\end{array}\right) \exp (\sigma t+i n \phi+i a z),
$$

where the perturbation amplitudes (quantities with hats) are assumed small. The complex growth rate $\sigma=\sigma_{r}+i \sigma_{i}$ determines marginal stability: the How is stable if $\sigma_{r}<0$ for all values of $n$ and $a$. If one sets $D=\partial / \partial r$, the linearized equations in the melt region $\eta /(1-\eta)<r<1 /(1-\eta)$ take the form

$$
\begin{gathered}
D \hat{u}+\frac{1}{r} \hat{u}+\frac{i n}{r} \hat{v}+i a \hat{w}=0 \\
\sigma \hat{u}+\frac{i n V}{r} \hat{u}+i a W \hat{u}+D \hat{p}= \\
\left(D^{2} \hat{u}+\frac{1}{r} D \hat{u}-\left[a^{2}+\frac{\left(1+n^{2}\right)}{r^{2}}\right] \hat{u}-\frac{2 i n}{r^{2}} \hat{v}\right)+2 \frac{(1-\epsilon \Theta) V}{r} \hat{v}-\frac{\epsilon V^{2}}{r} \hat{\Theta},
\end{gathered}
$$




$$
\begin{aligned}
& \sigma \hat{v}+\frac{i n V}{r} \hat{v}+i a W \hat{v}+\hat{u} D V+\frac{i n}{r} \hat{p}= \\
& \left(D^{2} \hat{v}+\frac{1}{r} D \hat{v}-\left[a^{2}+\frac{\left(1+n^{2}\right)}{r^{2}}\right] \hat{v}+\frac{2 i n}{r^{2}} \hat{u}\right)-\frac{(1-\epsilon \Theta) V}{r} \hat{u} \\
& \sigma \hat{w}+\frac{i n V}{r} \hat{w}+i a W \hat{w}+\hat{u} D W+i a \hat{p}= \\
& \left(D^{2} \hat{w}+\frac{1}{r} D \hat{w}-\left[a^{2}+\frac{n^{2}}{r^{2}}\right] \hat{w}\right)+G \hat{\Theta} \\
& \sigma \hat{\Theta}+\frac{i n V}{r} \hat{\Theta}+i a W \hat{\Theta}+\hat{u} D \Theta=\frac{1}{P_{r}}\left(D^{2} \hat{\Theta}+\frac{1}{r} D \hat{\Theta}-\left[a^{2}+\frac{n^{2}}{r^{2}}\right] \hat{\Theta}\right),
\end{aligned}
$$

and, in the region $\eta_{S} /(1-\eta)<r<\eta /(1-\eta)$, one obtains

$$
\sigma \hat{\Theta}_{S}+i n \operatorname{Re} \hat{\Theta}_{S}=\frac{1}{P_{s}}\left(D^{2} \hat{\Theta}_{S}+\frac{1}{r} D \hat{\Theta}_{S}-\left[a^{2}+\frac{n^{2}}{r^{2}}\right] \hat{\Theta}_{S}\right) .
$$

Here $P_{r}=\nu / \kappa_{L}$ is the Prandtl number, $P_{s}=\nu / \kappa_{S}$, and $\epsilon=\alpha \Delta T$.

The linearized boundary conditions are $\hat{u}=\hat{v}=\hat{w}=\hat{\Theta}=0$ at $r=$ $1 /(1-\eta), \hat{\Theta}_{S}=0$ at $r=\eta_{S} /(1-\eta)$, and, at the interface $r=R_{1}=\eta /(1-\eta)$,

$$
\begin{gathered}
\hat{u}=0, \\
\hat{w}+\hat{h} D W=0, \\
\hat{v}+\hat{h} D V=\operatorname{Re} \hat{h}, \\
\hat{\Theta}+\hat{h} D \Theta=\hat{\Theta}_{S}+\hat{h} D \Theta_{S}=-\gamma\left(a^{2}+\frac{\left(n^{2}-1\right)}{R_{1}^{2}}\right) \hat{h}, \\
-\sigma \mathcal{L} \hat{h}=\left(D \hat{\Theta}-q D \hat{\Theta}_{S}\right) .
\end{gathered}
$$

where $\gamma=\left(\bar{T}_{m} \Gamma\right) /(\bar{L} \Delta T), \mathcal{L}=\left(\nu L_{V}\right) /\left(k_{L} \Delta T\right)$, and $q=k_{S} / k_{L}$.

\section{Numerical Results}

The linearized equations constitute an eigenvalue problem from which the complex growth rate $\sigma=\sigma_{r}+i \sigma_{i}$ can be determined for each choice of the 
remaining parameters. Curves of marginal stability may be obtained by setting $\sigma_{r}=0$ and computing instead a pair $\left(\operatorname{Re}, \sigma_{i}\right)$ as a function of wavenumber, which can be done (see [20]) in the manner suggested by Keller [32]. The numerical procedure was checked carefully against previously published linear stability results for related problems [22, 27, 28, 33].

To illustrate the relative importance of the effects of buoyancy on the stability of the system, we use the material properties of succinonitrile [21] for a typical container geometry. We take $\bar{R}_{2}=1.60 \mathrm{~cm}, \bar{R}_{1}=1.11 \mathrm{~cm}$, and $\bar{R}_{0}=0.458 \mathrm{~cm}$, giving $\eta=0.690$ and $\eta_{S}=0.286$. We assume a temperature gradient in the melt of $2.5 \mathrm{~K} / \mathrm{cm}$, which produces a temperature difference across the melt gap of $\Delta T=1.225 \mathrm{~K}$, giving $\mathcal{L}=455, \epsilon=9.923 \cdot 10^{-4}$, $G=170$, and $\gamma=4.9 \cdot 10^{-3}$; for simplicity we set $\gamma=0$. The thermal properities of the liquid and solid are similar for succinonitrile, and we take the ratio of thermal conductivities to be $q=1$, with $P_{r}=P_{s}=22.8$. We restrict our discussion to axisymmetric disturbances $(n=0)$, which are expected to be the most dangerous modes in this configuration.

Marginal stability curves $\left(\sigma_{r}=0\right)$ are compared in Fig. 2 for four different cases. The Reynolds number Re of the flow is plotted versus the axial wavenumber $a$ of the disturbance. The curves in this plot are computed with $\epsilon=0$, so that the interaction of the density gradient with the centripetal acceleration is neglected.

The top two curves correspond to the classical Taylor-Couette problem with rigid isothermal surfaces bounding the liquid, and the bottom two curves correspond to the two-phase problem with a crystal-melt interface at $\bar{r}=\bar{R}_{1}$. We first consider the two cases without buoyancy $(G=0)$. The rigid-walled system has a critical Reynolds number $\mathrm{Re}=35$ (see Table I), corresponding to the onset of the classical secondary flow consisting of toroidal Taylor-vortex cells. The crystal-melt interface destabilizes the system, giving a smaller critical Reynolds number $\operatorname{Re}=9$; the axial wave- 
length of the most dangerous disturbance, $\lambda=2 \pi / a$, is shifted to larger values. This effect has been described in our previous work [22, 23]; the size of the destabilization is found to vary with the Prandtl number of the melt. The results for a rigid-walled system actually provide the limiting values for a conduction-dominated system with $P_{r} \ll 0$. The destabilization due to a crystal-melt interface becomes pronounced for convection-dominated systems with $P_{r} \gg 1$. For $G=0$ the disturbances are stationary in both cases, with $\sigma_{i}=0$.

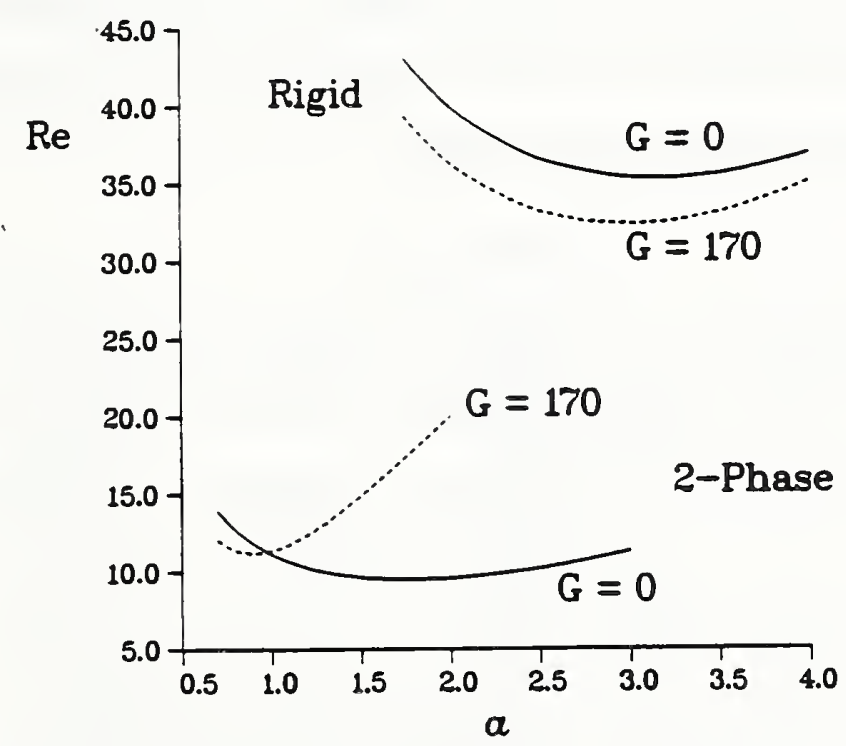

FIG. 2. Marginal values of the Reynolds number, Re, versus the axial wavenumber of the perturbation, $a$, for axisymmetric disturbances, comparing the effects of buoyancy $(G=170)$ for the rigid-walled system (top two curves) and for the system with a crystalline inner cylinder (lower two curves).

For $G=170$ the marginal curves are shifted, as indicated by the dashed curves in Fig. 2. The rigid-walled system is destabilized slightly from $\operatorname{Re}=35$ to $R e=32$, with a small increase in wavelength. This destabilization of the Taylor-Couette flow by the buoyancy forces is consistent with the trends reported previously [27]. The toroidal cells are no longer stationary, but drift downwards with an axial phase velocity equal to $-\sigma_{i} / a$. The effect of buoyancy when a crystal-melt interface is present is quite different, 
however. There is a slight increase in the critical Reynolds number when buoyancy is present, and there is a two-fold increase in the wavelength of the most dangerous disturbance. The secondary cells have a slow upward drift $\left(\sigma_{i}<0\right)$, but the phase velocity is smaller by three orders of magnitude. This decrease in phase velocity is likely due to the strong coupling between the interface and the flow: the interface deforms and accommodates the cellular flow structure, allowing the instability to occur at lower Reynolds numbers. For the cellular structure to translate in the axial direction, however, the deformed interface must melt and freeze for the wave to propagate, which retards the dynamics of the process.

\section{Table I}

\begin{tabular}{cccccc}
\hline$P_{\mathrm{r}}$ & $G$ & $\epsilon$ & $\mathrm{Re}$ & $\sigma_{i}$ & $a$ \\
\hline 0 & 0 & 0 & 35.2520 & 0 & 3.143 \\
0 & 0 & 0.001 & 35.3777 & 0 & 3.143 \\
0 & 170 & 0 & 32.3174 & 4.6937 & 2.967 \\
0 & 170 & 0.001 & 32.2991 & 4.6881 & 2.967 \\
22.8 & 0 & 0 & 9.4518 & 0 & 1.782 \\
22.8 & 0 & 0.001 & 9.4508 & 0 & 1.782 \\
22.8 & 170 & 0.0 & 11.1517 & $-2.7096 \cdot 10^{-3}$ & 0.904 \\
22.8 & 170 & 0.001 & 11.1509 & $-2.7101 \cdot 10^{-3}$ & 0.904 \\
\hline
\end{tabular}

Critical values of the Reynolds number $R e$, the time constant $\sigma_{i}$, and the critical wavenumber $a$ for the rigid-walled case $\left(P_{r}=0\right)$ and the case of a crystalline inner annulus of succinonitrile $\left(P_{r}=22.8\right)$. Buoyancy forces are absent for $G=0$, and the interaction of the centripetal acceleration with the radial density is absent for $\epsilon=0$.

In Table I we give values of the critical Reynolds numbers, the time constant $\sigma_{i}$, and the critical wavenumber $a$ for the cases shown in Fig. 2 , and we also give results for the same cases computed with a non-zero contribution from the interaction of the centripetal acceleration with the radial density gradient, using the value $\epsilon=0.001$. The changes brought about by including density variation in the centripetal acceleration are seen to be insignificant for these cases. The larger change in the stability of the 
system with a radial temperature gradient is clearly due to gravitational forces.

\section{Acknowledgements}

This work was conducted with the support of the Microgravity Science and Applications Division of the National Aeronautics and Space Administration, and the Applied and Computational Mathematics Program of the Defense Advanced Research Projects Agency. One of the authors (BTM) was supported by an NRC Postdoctoral Research Fellowship.

\section{References}

[1] D. T. J. Hurle and E. Jakeman, Introduction to the techniques of crystal growth, PCH PhysicoChemical Hydrodynamics 2 (1981) pp. 237-244.

[2] R. A. Brown, Theory of transport processes in single crystal growth from the melt, AIChE J. 34 (1988) pp. 881-911.

[3] S. R. Coriell, G. B. McFadden and R. F. Sekerka, Cellular growth during directional solidification, Ann. Rev. Mater. Sci. 15 (1985) pp. 119-145.

[4] M. E. Glicksman, S. R. Coriell and G. B. McFadden, Interaction of flows with the crystal-melt interface, Ann. Rev. Fluid Mech. 18 (1986) pp. 307-335.

[5] W. W. Mullins and R. F. Sekerka, Stability of a planar interface during solidification of a dilute binary alloy, J. Appl. Phys. 35 (1964) pp. 444451.

[6] S. R. Coriell and R. F. Sekerka, Effect of convective flow on morphological stability, PCH PhysicoChem. Hydrodyn. 2 (1981) pp. 281-293.

[7] S. R. Coriell, M. R. Cordes, W. J. Boettinger, and R. F. Sekerka, Convective and interfacial instabilities during unidirectional solidification of a binary alloy, J. Crystal Growth 49 (1980) pp. 13-28.

[8] M. E. Glicksman, E. Winsa, R. C. Hahn, T. A. Lograsso, S. H. Tirmizi, and M. E. Selleck, Isothermal dendritic growth - a proposed microgravity experiment, Metall. Trans. 19A (1988) pp. 1945-1953. 
[9] S. H. Davis, Hydrodynamic interactions in directional solidification, J. Fluid. Mech. 212 (1990) pp. 241-262.

[10] R. T. Delves, Theory of Interface Stability, in Crystal Growth, B. R. Pamplin, ed., Pergamon, Oxford, 1974, pp. 40-103.

[11] S. R. Coriell, G. B. McFadden, R. F. Boisvert, and R. F. Sekerka, Effect of a forced Couette flow on coupled convective and morphological instabilities during unidirectional solidification, J. Crystal Growth 69 (1984) pp. 15-22.

[12] S. R. Coriell and G. B. McFadden, Buoyancy effects on morphological instability during directional solidification, J. Crystal Growth 94 (1989) pp. 513-521.

[13] K. Brattkus, and S. H. Davis, Flow induced morphological instability: stagnation point flows, J. Crystal Growth 89 (1988) pp. 423-427.

[14] G. B. McFadden, S. R. Coriell, and J. I. D. Alexander, Hydrodynamic and free boundary instabilities during crystal growth: the effect of a plane stagnation flow, Comm. Pure and Appl. Math 41 (1988) pp. 683-706.

[15] K. Brattkus and S. H. Davis, Flow induced morphological instability: the rotating disk, J. Crystal Growth 87 (1988) pp. 385-396.

[16] S. A. Forth and A. A. Wheeler, Hydrodynamic and morphological stability of the unidirectional solidification of a freezing binary alloy: a simple model, J. Fluid Mech. 202 (1989) pp. 339-366.

[17] S. H. Davis, U. Müller, and C. Dietsche, Pattern selection in singlecomponent systems coupling Bénard convection and solidification, J. Fluid. Mech. 144 (1984) pp. 133-151.

[18] B. Caroli, C. Caroli, C. Misbah, and B. Roulet, Solutal convection and morphological instability in directional solidification of binary alloys, $\mathrm{J}$. Phys. (Paris) 46 (1985) pp. 401-413.

[19] G. W. Young and S. H. Davis, Directional solidification with buoyancy in systems with small segregation coefficient, Phys. Rev. B34 (1986) pp: 3388-3396.

[20] G. B. McFadden, S. R. Coriell, R. F. Boisvert, M. E. Glicksman, and Q. T. Fang, Morphological stability in the presence of fluid flow in the melt, Metall. Trans. 15A (1984) pp. 2117-2124. 
[21] Q. T. Fang, M. E. Glicksman, S. R. Coriell, G. B. McFadden, and R. F. Boisvert, Convective influence on the stability of a crystal-melt interface, J. Fluid Mech. 151 (1985) pp. 121-140.

[22] G. B. McFadden, S. R. Coriell, M. E. Glicksman, and M. E. Selleck, Instability of a Taylor-Couette flow interacting with a crystal-melt interface, PhysicoChem. Hydrodyn. 11 (1989) pp. 387-409.

[23] G. B. McFadden, S. R. Coriell, B. T. Murray, M. E. Glicksman, and M. E. Selleck, Effect of a crystal-melt interface on Taylor-vortex flow, Phys. Fluids A 2 (1990) pp. 700-705.

[24] S. K. F. Karlsson and H. A. Snyder, Observations on a thermally induced instability between rotating cylinders, Ann. Phys. 31 (1965) pp. 314-324.

[25] K. S. Ball, B. Farouk, and V. C. Dixit, An experimental study of heat transfer in a vertical annulus with a rotating inner cylinder, Int. J. Heat Mass Transfer 32 (1989) pp. 1517-1527.

[26] K. S. Ball and B. Farouk, Bifurcation phenomena in Taylor-Couette flow with buoyancy effects, J. Fluid Mech. 197 (1988) pp. 479-501.

[27] M. E. Ali, The stability of Taylor-Couette flow with radial heating. Ph. D. Dissertation, Dept. of Mech. Eng., University of Colorado, Boulder, Colorado, 1988.

[28] J. Walowit, S. Tsao, and R. C. DiPrima, Stability of flow between arbitrarily spaced concentric cylindrical surfaces including the effect of a radial temperature gradient, Trans. Am. Soc. Mech. Eng. E: J. Appl. Mech. 31 (1964) pp. 585-592.

[29] G. I. Taylor, Phil. Trans. Roy. Soc. A 223, 289 (1923).

[30] P. G. Drazin and W. H. Reid, Hydrodynamic Stability, Cambridge University Press, 1981.

[31] S. Chandrasekhar, Hydrodynamic and Hydromagnetic Stability, Oxford University Press, 1961.

[32] H. B. Keller, Numerical Solution of Two Point Boundary Value Problems, Regional Conference Series in Applied Mathematics 24, SIAM, Philadelphia, 1976.

[33] G. B. McFadden, S. R. Coriell, R. F. Boisvert, and M. E. Glicksman, Asymmetric instabilities in buoyancy-driven flow in a tall vertical annulus, Phys. Fluids 27 (1984) pp. 1359-1361. 



\begin{tabular}{|c|c|c|}
\hline \multirow[t]{4}{*}{$\begin{array}{l}\text { NIST-114A } \\
\text { (REV. 3-89) }\end{array}$} & \multirow{4}{*}{$\begin{array}{l}\text { U.S. DEPARTMENT OF COMMERCE } \\
\text { NATIONAL INSTITUTE OF STANDARDS AND TECHNOLOGY } \\
\text { BIBLIOGRAPHIC DATA SHEET }\end{array}$} & $\begin{array}{l}\text { 1. PUBUCATION OA REPORT NUMBEF } \\
\text { NISTIR } 4364\end{array}$ \\
\hline & & 2. PERFORMING ORGANIZATION REPORT NUMBER \\
\hline & & \\
\hline & & JuIY 1990 \\
\hline
\end{tabular}

4. TITLE AND SUBTITLE

Effect of a Crystal-Melt Interface on

Taylor-Vortex Flow with Buoyancy

5. AUTHOR(S)

G. B. McFadden, B. T. Murray, S. R. Coriell, M. E. Glicksman, and M. E. Selleck

\begin{tabular}{l|l|l}
\hline 6. PERFORMING ORGAMIZATION (IF JOINT OR OTHER THAN MIST, SEE INSTRUCTIONS) & 7. CONTRACT/GAANT NUMBER
\end{tabular}

U.S. DEPARTMENT OF COMMERGE

NATIONAL INSTITUTE OF STANDARDS AND TECHNOLOGY

GAITHERSBUPG, MD 20899

8. TYPE OF REPOAT AND PEAIOD COVERED

Final

9. SPONSORING ORGANIZATION NAME AND COMPLETE ADDRESS (STAEET, CITY, STATE, ZIP)

Same as item 6.

10. SUPPLEMENTARY NOTES

DOCUMENT DESCAIBES A COMPUTER PROGRAM; SF-185, FIPS SOFTWARE SUMMARY, IS ATTACHED.

11. ABSTRACT (A 200-WORD OR LESS FACTUAL SUMMARY OF MOST SIGNIFICANT INFORMATION. IF DOCUMENT INCLUDES A SIGNIFICANT BIBLOGRAPHY OR LITERATURE SURVEY, MENTION IT HERE.)

During crystal growth from the liquid, a fundamental problem is to understand the interaction of the crystal-melt interface with fluid flow in the liquid. This problem combines the complexities of the Navier-Stokes equations for fluid flow in the liquid with the nonlinear behavior of the free boundary representing the crystal-melt interface. Some progress has been made by studying explicit flows that allow a base state corresponding to a one-dimensional crystal-melt interface with solute and/or temperature fields that depend only on the distance from the interface. This allows the strength of the interaction between the flow and the interface to be assessed by a linear stability analysis of the simple base state. The case of a Taylor-Couette flow interacting with a cylindrical crystalline interface is currently being investigated both experimentally and theoretically. We consider the changes in the linear stability of this system produced by density-driven convection generated by the interaction of the density gradients with the gravitational and centripetal acceleration.

12. KEY WOADS (6 TO 12 ENTAIES; ALPHABETICAL ORDEA; CAPITALZE ONLY PROPER NAMES; AND SEPARATE KEY WOADS BY SEMICOLONS)

Tzylor-Couette flow; crystal-melt interface; linear stability; solidification;

hydrodynamic stability; flow-interface interactions

13. AVAILABILITY

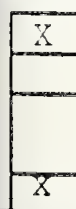

UNLIMITED

FOR OFFICIAL. DISTRIBUTION. DO NOT RELEASE TO NATIONAL TECHNICAL INFORMATION SEAVICE (NTIS).

ORDER FROM SUPERINTENDENT OF DOCUMENTS, U.S. GOVERNMENT PRINTING OFFICE, WASHINGTON, DC 20402.

ORDER FROM NATIONAL TECHMICAL INFORMATION SERVICE (NTIS), SPAINGFIELD, VA 22161.

\begin{tabular}{l} 
14. NUMBER OF PRINTED PAGES \\
18 \\
\hline 15. PRICE $\mathrm{AO} 2$ \\
\hline
\end{tabular}



